OPEN ACCESS

Edited by:

Nino Russo,

University of Calabria, Italy

Reviewed by:

Piercarlo Fantucci,

University of Milano-Bicocca, Italy

Cina Foroutan-Nejad,

Masaryk University, Czechia

*Correspondence:

Nathália M. P. Rosa

nathympaixao@yahoo.com.br

Luiz Antônio S. Costa

luiz.costa@uff.edu.br

Specialty section:

This article was submitted to

Theoretical and Computational

Chemistry,

a section of the journal

Frontiers in Chemistry

Received: 06 February 2019

Accepted: 18 April 2019

Published: 21 May 2019

Citation:

Rosa NMP, Ferreira FHC, Farrell NP and Costa LAS (2019) TriplatinNC and Biomolecules: Building Models Based

on Non-covalent Interactions.

Front. Chem. 7:307.

doi: 10.3389/fchem.2019.00307

\section{TriplatinNC and Biomolecules: Building Models Based on Non-covalent Interactions}

\author{
Nathália M. P. Rosa ${ }^{1 *}$, Frederico Henrique do C. Ferreira ${ }^{1}$, Nicholas P. Farrell ${ }^{2}$ and \\ Luiz Antônio S. Costa ${ }^{1 *}$ \\ ' Núcleo de Estudos em Química Computacional, Departamento de Química, ICE, Universidade Federal de Juiz de Fora, Juiz \\ de Fora, Brazil, ${ }^{2}$ Department of Chemistry, Virginia Commonwealth University, Richmond, VA, United States
}

The class of polynuclear platinum(II) compounds have demonstrated a great interest because their high activity against cancer cells. Among these new compounds, the TriplatinNC also called AH78, demonstrated surprising antitumor activity, in some cases equivalent to cisplatin. It is well-known that complex charge +8 favors interaction with DNA and other biomolecules non-covalently, through the hydrogen bonds with phosphate and sulfate groups present in these structures. The hydrogen atoms of the amine interact with the oxygen atoms of the phosphate and sulfate groups present in the DNA strand and heparan sulfate, respectively. These interactions can cause significant twists in double helix and inhibit the activity of these biomolecules. The present investigation is an attempt to provide a benchmark theoretical study about TriplatinNC. We have described the non-covalent interactions through small reliable mimetic models. The non-covalent interactions were also evaluated on larger models containing DNA fractions with six nitrogenous base pairs (CGCGAA) and fractions of the disaccharide that makes the HS evaluated by the hybrid QM/MM ONIOM methodology.

Keywords: triplatin complexes, biomolecules, phosphate clamps, sulfate clamps, arginine-forks, mimetic model, DFT, ONIOM

\section{INTRODUCTION}

Cisplatin has been the most widely metal-based drug used for the treatment of cancer in almost four decades, but the efficacy of this drug has become more difficult because of acquired resistance and severe side effects such as nephrotoxicity, neurotoxicity, and hearing system damage (Wong and Giandomenico, 1999). Other platinum complexes have been approved for clinical use worldwide, such as carboplatin (second generation), oxaliplatin (third generation), and three compounds as nedaplatin, lobaplatin, and heptaplatin, which have been approved only in Japan, China, and South Korea, respectively (Barry and Sadler, 2013). Therefore, approaches that have a different cellular response from that of cisplatin have been the target of studies in several areas.

The searching for new compounds such as polynuclear platinum complexes (PPCs) that show anti-cancer activity have increase in recent years (Mangrum and Farrell, 2010; Prisecaru et al., 2014; Farrell, 2015; Qu et al., 2015). PPCs have shown great promise against cancer cells due to faster and more effective interactions with the DNA when compared to mononuclear complexes because they have more than one platinum core available to coordinate, exhibiting chemical and biological properties that differ significantly to cisplatin (Qu et al., 2004). 


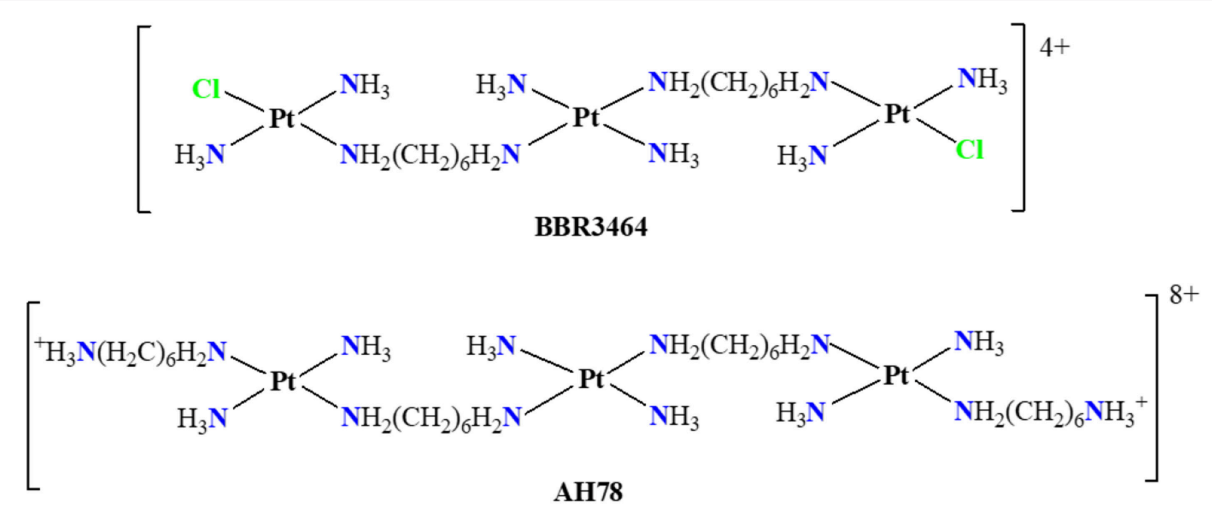

FIGURE 1 | Planar structures of trinuclear platinum(II) complexes, Triplatin (BBR3464), and TriplatinNC (AH78).

The Triplatin complex BBR3464 (Figure 1) entered phase I of clinical trials in the 1990s and was the first multinuclear platinum compound to enter in phase II (Olszewski and Hamilton, 2010; $\mathrm{Qu}$ et al., 2015). Two of the three platinum centers in the Triplatin have chloride ligands, which allows the formation of two mono-functional adducts with DNA by long range crosslinks (Manzotti et al., 2000; Boulikas and Vougiouka, 2003). The Triplatin (BBR3464) has demonstrated biological activity with cytotoxicity at concentrations in the micromolar order, similarly to cisplatin for some human cancer cell lines, they also have shown great potential to overcome the effects of drug resistance, and in many cases the solubility in water is appreciably the which makes it favorable for clinical practice (Riccardi et al., 2001; Wheate and Collins, 2005; Benedetti et al., 2011).

In principle, it was believed that platinum complexes should be neutral in order to exhibit anti-tumor activity. PPCs challenge this paradigm by presenting different forms of interaction with DNA. The TriplatinNC (Figure 1) complex shows overall charge +8 , presenting cellular uptake about 5 times greater than its analog Triplatin, which has overall charge +4 and is comparable to that of cisplatin in some cell lines (Harris et al., 2005).

PPCs have different coordination modes to the DNA, inaccessible to mononuclear complexes; in mononuclear complexes short range connections, of type 1,2-intrastrand with DNA are predominant. Polynuclear platinum (II) complexes are capable of forming long-range coordination such as DNA inter- and intra-strand links (Komeda et al., 2010). These links can occur with distances up to 4 pairs of nitrogenous bases (Kasparkova et al., 2002). The charge created by the metal centers increases the binding kinetics with the DNA, because the connection is achieved by two coordination spheres of independent mono-functional platinum, eliminating many problems of steric hindrance which are present when two nucleobases are attached to one Pt atom, such as for cisplatin and its analogs. The charge present in the complex also impacts the extent and nature of the inter- and intra-strand crosslinks (Hegmans et al., 2004; Qu et al., 2015). Malina et al. have reported that the presence of the charged structure, as in the TriplatinNC, greatly can condense DNA and inhibit polymerase activity (Malina et al., 2014).
Differently from cisplatin, the trinuclear platinum (II) complexes shown in Figure 1 do not interact covalently with the DNA because they have no leaving groups, such as chloride present in the cisplatin. The observed non-covalent interactions are mainly of electrostatic type and the hydrogen bonds have an important role causing major distortions in the DNA double helix (Mangrum and Farrell, 2010). Komeda et al. (2006) report the crystal structure (PDB:2DYW) where the complex forms hydrogen bonds with the oxygen atoms of phosphate groups present in DNA (Mangrum and Farrell, 2010). These interactions occur between the hydrogen atoms of the amino groups of the complex and the oxygen atoms of the double helix backbone phosphates of DNA. Such interactions are known as phosphate clamps and may be backbone tracking or minor groove spanning. This term is used to indicate a cyclic structure in which one phosphate interacts with two hydrogen atoms from two $\mathrm{a}(\mathrm{m})$ mine ligands bound to a platinum core (Komeda et al., 2006; Wang and Gao, 2014). The exact mode depends on the specificity of base or helical local topological parameters of the oligonucleotide (Prisecaru et al., 2014).

Recent studies have shown another likely target for PPCs, glycosaminoglycans (GAGs), such as heparan sulfate (HS), which are linear polysaccharides composed of repeated disaccharide units of alternating residues of uronic acid and hexosamine. These molecules are directly related to the processes of angiogenesis and metastasis and when conjugated to proteins are found on the cell surface and extracellular matrix with critical functions in cell adhesion and migration. The TriplatinNC complex was shown to be capable of inhibiting the activity of these biomolecules by the formation of non-covalent interactions, through inhibition of Growth Factor Binding to HS, such as sulfate clamps, which are structures similar to those of phosphate clamps. The conformational similarity between $\mathrm{HS}$ and DNA justifies the choice of these targets for this study (Peterson et al., 2017; Katner et al., 2018).

Another possible structure is the phosphate or sulfate arginine-forks characterized by the formation of an eightmembered ring (including hydrogen atoms) stabilized by two hydrogen bonds between two a $(\mathrm{m})$ mine groups at the 
cis-positions that interact with two oxygen atoms from the phosphate or sulfate. The $\mathrm{PtN}_{4}$ type of Platinum (II) centers have high selectivity for oxygen atoms present in biomolecules such as DNA and HS, being prone to the formation of these structures that have highly conserved geometry of binding (Komeda et al., 2010). Peterson and colleagues recently demonstrated that TriplatinNC is capable of blocking the interaction of different proteins with both DNA and HS (Peterson et al., 2014, 2017). The platinum (II) trinuclear complex inhibits the formation of the interaction between the TBP protein and DNA (Peterson et al., 2014).

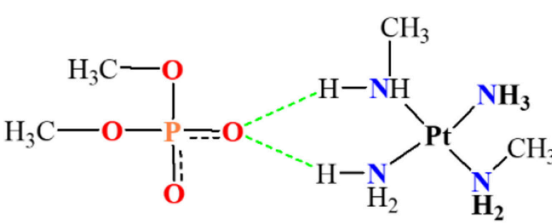

Phosphate Clamp<smiles>CN[P+](N)(NC)[In]OS(=O)(=O)OC</smiles>

Sulfate Clamp<smiles>CN[P+](N)(NC)N[IH][CH]N</smiles>

Phosphate Arginine-fork<smiles>CN[P+](N)(NC)N[IH][CH]OS(=O)(=O)OC</smiles>

Sulfate Arginine-fork

FIGURE 2 | Mimetic models of phosphate and sulfate clamps and arginine-forks.
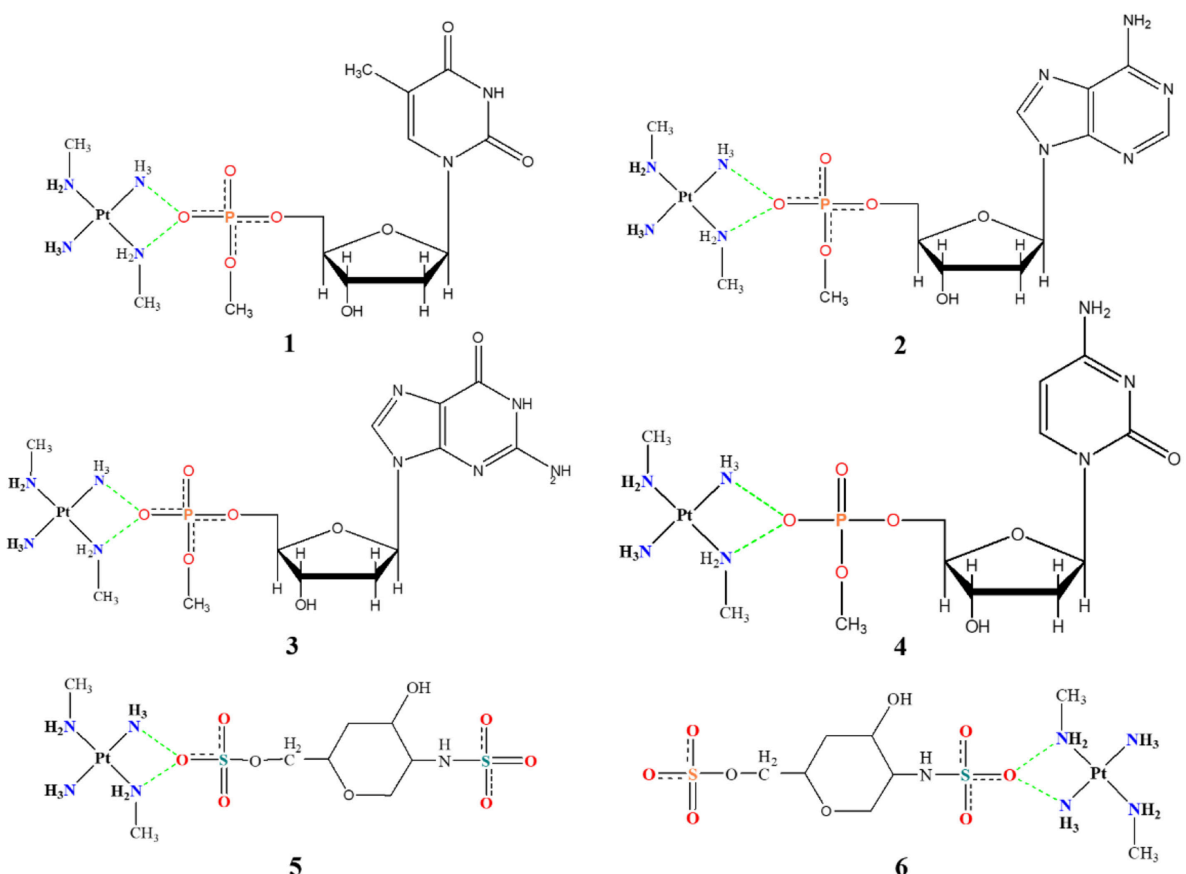

5

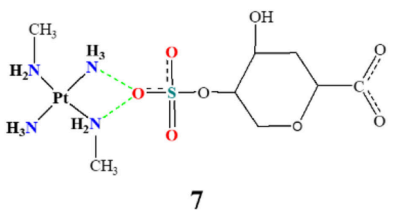

FIGURE 3 | Mimetic models based on nitrogenous bases and fractions of the disaccharide. 

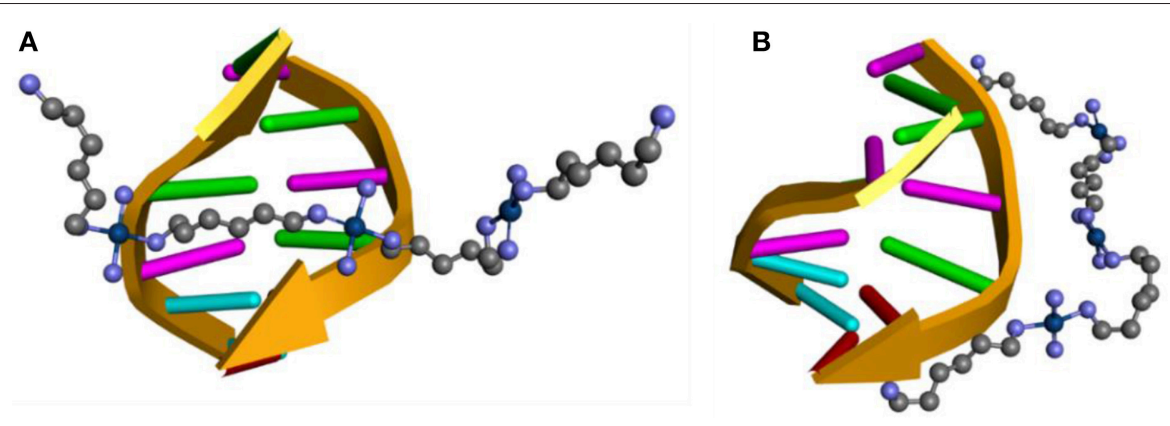

FIGURE 4 | Scheme of the interaction position between the TriplatinNC complex with DNA: (A) Model A, (B) Model B.

TABLE 1 | Energy values of Gibbs free energy of interaction in kcal mol ${ }^{-1}$.

\begin{tabular}{lccllr}
\hline$\Delta \mathbf{E}_{\text {int }}$ & \multicolumn{2}{c}{ B3LYP } & & & \multicolumn{2}{c}{ BHandH } \\
\cline { 2 - 3 } \cline { 5 - 6 } & Phosphate & Sulfate & & Phosphate & Sulfate \\
\hline Clamp & -2.28 & 3.45 & & -11.11 & -6.12 \\
Arginine-Fork & -3.63 & 2.06 & & -12.79 & -8.29 \\
\hline
\end{tabular}

The use of computational hybrid methods has been a very successful approach to enable more accurate performance on relatively large chemical systems combining quantum mechanics (QM) and molecular mechanics (MM) (Heerdt and Morgon, 2011). The already well-known method called ONIOM (Our Own n-layered Integrated Molecular Orbital and Molecular Mechanics), developed by Morokuma (2003), is a method that combines a variety of quantum methods with a multi-layered molecular mechanics method. The ONIOM method has been successfully applied in the study of interactions between DNA and metal complexes (Gkionis and Platts, 2012; Gkionis et al., 2013; Chung et al., 2015). These interactions, on several occasions, influence the cytotoxicity of the drug and can affect the DNA repair and other processes.

In recent years, although most of the theoretical studies of platinum drugs mostly involve the complex reaction mechanism with the purine bases of DNA, some theoretical studies have investigated on the PPC's reaction mechanism as anticancer drugs and their interaction with biological target. Chen and Zhou (2015) studied the mono-functional substitution reactions between cisplatin and PPCs, as Triplatin (BBR3464) with sulfur biomolecules and purine bases (guanine and adenine) in DNA with two different functional methods DFT-B3LYP/M06 by solvation model IEF-PCM. Recently, our group reported different works on platinum compounds involving the study of the hydrolysis process of a platinum dinuclear complex (Esteves et al., 2015), as well as inclusion complexes of binuclear platinum (II) complex (bisPt) into $\alpha-, \beta-, \quad$ and $\gamma$-cyclodextrins via hydrogen interactions formed preferably by hydrogen atoms present in bisPt and the oxygen atoms from inside the cyclodextrin molecules (Paixão et al., 2012).
This study aims to describe the non-covalent interactions with TriplatinNC with DNA and HS complex through small reliable mimetic models, and by the use of DNA fractions containing six pairs of nitrogenous bases.

\section{METHODOLOGY}

One of the problems in the study of many atoms systems is the "how" to diminish the size of the system while keeping the properties that might be of interest. The use of smaller chemical models that can represent the site of interest, in a feasible time, is an important strategy of study. The existing hydrogen interactions between the platinum centers and the DNA bases and HS were studied here by mimetic models. Initially, mimetic models were studied in order to evaluate the interaction energies for the structures of phosphate and sulfate clamps and arginine-forks, according to the models shown in Figure 2. These models were evaluated using the B3LYP (Becke, 1993a) and BHandH (Becke, 1993b) functional with the pseudopotential LANL2DZ (Hay and Wadt, 1985a,b,c) and SDD (Andrae et al., 1990), respectively for the platinum atom as well as the $6-31+\mathrm{G}(\mathrm{d}, \mathrm{p})$ (Here et al., 1972) basis set to the other atoms.

Another seven models have been developed and are shown in Figure 3. Models 1-4 consist of the platinum coordination sphere and the nucleotide where the nitrogenous bases are thymine $(\mathrm{T})$, adenine $(\mathrm{A})$, guanine $(\mathrm{G})$, and cytosine $(\mathrm{C})$, respectively, and all atoms remained free. The models 5-7 consist of the platinum coordination sphere and fractions of the disaccharide that make the HS. These models were evaluated using the BHandH functional with the pseudopotential SDD for the platinum atom as well as the $6-31+G(d, p)$ basis set to the other atoms. All models have been optimized for clamps and arginine-forks structures. Natural Bond Orbital (NBO) calculations have also been performed at the very same level of theory described above.

The ONIOM hybrid method was used to evaluate the noncovalent interactions between a DNA fragment composed of 6 nitrogenous base pairs (CGCGAA) and the TriplatinNC complex arranged in two different ways, one with the complex spanning the two DNA strands, Model A (Figure 4A) and another with the complex placed along the same ribbon of the DNA fragment, 
Model B (Figure 4B). These structures were based on results reported by Komeda et al. (2010) and Prisecaru et al. (2014). The ONIOM method was also used to evaluate the interactions between the sulfate groups of a model of heparan sulfate formed by 3 units of the HS disaccharide, which corresponds to the half of the model reported in the PDB database, with 1HPN code and the TriplatinNC complex, Model C (Mulloy et al., 1993). The structures were obtained by defining for the upper layer the functional $\mathrm{BHand} \mathrm{H}$, with the set of basic functions $6-31+\mathrm{G}(\mathrm{d}, \mathrm{p})$ for the non-metallic atoms and SDD for the platinum atoms. For the low layer the force field UFF is being used (Rappé et al., 1992). Sodium atoms were added close to the phosphate groups that are not involved in the formation of phosphate clamps to decrease the loads of each layer. All the structures of the complexes were completely optimized using the IEFPCM model (Scalmani and Frisch, 2010) to include the solvent continuously, considering the molecule of the solute inserted in a medium of dielectric constant equal to that of water $\left(\varepsilon=78,35^{*}\right)$. All calculations, including $\mathrm{NBO}$, were performed using the program package GAUSSIAN09 (Frisch et al., 2016).

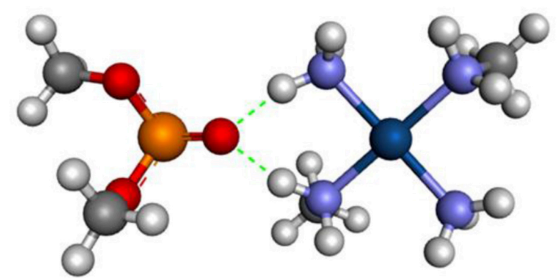

Phosphate Clamp

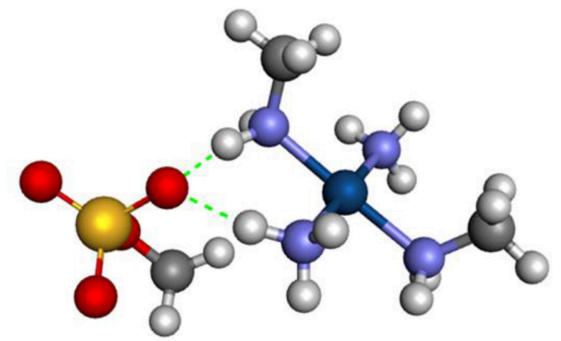

Sulfate Clamp

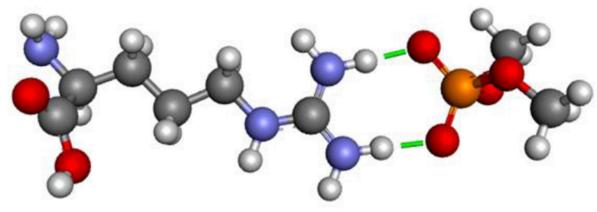

Arginine-fork $\cdots$ phosphate

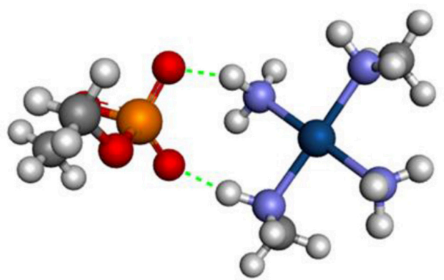

Phosphate Arginine-fork

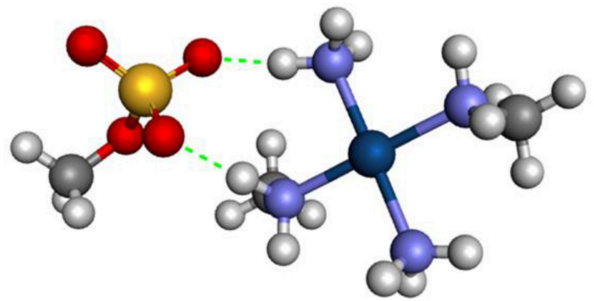

Sulfate Arginine-fork

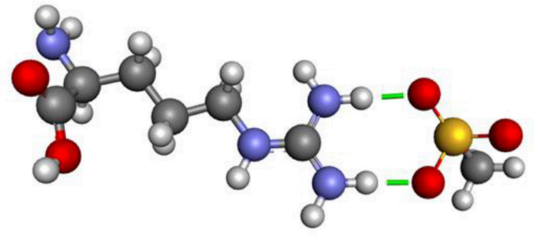

Arginine-fork $\cdots$ sulfate

FIGURE 5 | Optimized structures of the models using the BHandH functional.

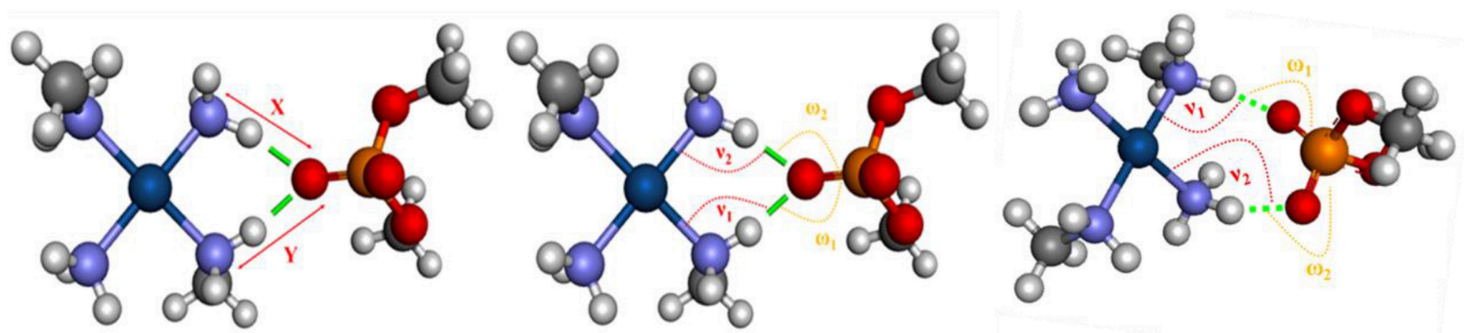

FIGURE 6 | Bond distances and angles analyzed in models. Refer to Table 2 to see the values of $x, y, v_{1}, v_{2}, \omega_{1}$, and $\omega_{2}$. 
TABLE 2 | Distances (in Ångström) and bond angles (in degree) involved in the formation of clamps and arginine-forks using the $\mathrm{BHandH}$ functional.

\begin{tabular}{|c|c|c|c|c|c|c|c|}
\hline & & \multicolumn{2}{|c|}{ Distances/Å } & \multicolumn{4}{|c|}{ Angles $/^{\circ}$} \\
\hline & & $\mathbf{x}$ & $\mathbf{y}$ & $v_{1}$ & $v_{2}$ & $\omega_{1}$ & $\omega_{2}$ \\
\hline \multirow[t]{2}{*}{ Phosphate } & Clamps & 2.70 & 2.70 & 100.6 & 100.7 & 129.5 & 138.0 \\
\hline & Arginine-forks & 2.74 & 2.69 & 103.4 & 111.7 & 127.0 & 96.3 \\
\hline \multirow[t]{2}{*}{ Sulfate } & Clamps & 2.75 & 2.76 & 101.2 & 101.7 & 130.6 & 128.5 \\
\hline & Arginine-forks & 2.78 & 2.78 & 112.4 & 118.2 & 108.1 & 123.7 \\
\hline \multirow[t]{2}{*}{ Arginine-Forks } & Phosphate & 2.67 & 2.68 & 122.9 & 122.6 & 117.7 & 117.4 \\
\hline & Sulfate & 2.74 & 2.75 & 120.4 & 121.6 & 121.1 & 120.5 \\
\hline
\end{tabular}

\section{RESULTS AND DISCUSSION}

Table 1 shows the values of interaction energies in kcal $\mathrm{mol}^{-1}$ for the models described in Figure 2. It is possible to observe that the structures obtained using the $\mathrm{BHandH}$ functional present the lowest free Gibbs energy values of interaction. Among these, it is noted that the arginine-fork structures present the most negative values, suggesting that such structures are more stable than the clamps, for the methodology studied. In order to compare, we analyzed a model that represents the arginine-forks composed of a phosphate group and the amino acid arginine (Figure 5). The Gibbs free energy values for the arginine-Forks are -7.88 and -7.38 $\mathrm{kcal} \mathrm{mol}^{-1}$ for the phosphate and sulfate groups, respectively, contributing to the assertion that from the analyzed models the arginine-forks type structures can be more stable, from the point of view of the free energy of Gibbs for the methodology studied.

Figure 5 shows the optimized structures obtained for the models analyzed with the respective hydrogen interactions among the hydrogen atoms from the amino groups of the platinum center with the oxygen atoms present in the DNA phosphate groups and HS sulfate groups using the $\mathrm{BHandH}$ functional.

According to Komeda et al. (2006), a phosphate clamp is a cyclic structure with a single oxygen atom of the phosphate group, which interacts via two hydrogen bonds from each of two amino ligands from a single $\mathrm{Pt}(\mathrm{II})$ center. It was observed that the formation of phosphate clamps seems to require the cis orientation of the nitrogenous ligands so that trans ligands do not participate in phosphate clamps, the mutually trans groups being too distant for the occurrence of interaction with a single phosphate group oxygen atom. Figure 6 shows the angles and distances analyzed and Table 2 presents the values obtained for the models of Figure 5.

The experimental values reported by Komeda et al. (2006) for $\mathrm{x}$ and $\mathrm{y}$ for the phosphate clamps are between 2.75 and 2.98 $\AA$ and between 2.83 and $3.22 \AA$, respectively. Thus, the values obtained for the mimetic models are close to the values reported in the literature. Considering that the structures formed by the sulfate groups are similar to those formed by the phosphate groups, it can be concluded that the values found for the binding distances for such structures are similar to the values reported in the literature to the phosphate clamps. For the analyzed angles, the experimental values for $\nu_{1}$ vary between 99 and $106^{\circ}$ and for $\nu_{2}$ between 88 and $109^{\circ}$, from these data it was observed that the values obtained were similar to the experimental data. For the angles $\omega_{1}$ the values found are close to the experimental data that are between 113 and $145^{\circ}$, but for the angle $\omega_{2}$ it was observed that some values are below the experimentally reported interval, where the experimental values are between 128 and $151^{\circ}$, however for the sulfate clamp, angle $\omega_{2}$ is very close to that reported in the literature for phosphate clamps. Comparing with the results obtained for the arginine-forks with the phosphate and sulfate groups, it is noted that the values for the distances are close to the values obtained with the non-covalent structures formed by the centers of platinum (II). With respect to the angles, $v_{1}$ and $v_{2}$ are smaller when formed between the center of platinum and the phosphate and sulfate groups, already the angles $\omega_{1}$ and $\omega_{2}$ are mostly larger when compared to the results obtained for the arginine-forks.

Figures 7, 8 show the structures optimized for the 1-4 and 5-7 models, respectively. It is possible to note the formation of non-covalent interactions in all the structures obtained. In the models $2,3,5$, and 7 we can observe the formation of both structures, phosphate clamps and phosphate arginineforks. Table 3 shows the interaction distances involved in the described structures. The mean values obtained for the distances $\mathrm{x}$ and $\mathrm{y}$ are below the mean values reported by Komeda et al. (2006). The values of $v_{1}$ and $v_{1}$ are above the experimental average values. The mean values of the angles $\omega_{1}$ and $\omega_{2}$ are smaller than the experimental data, this is due to the contribution of the results obtained for the arginineforks that presents a structure in which two oxygen atoms interact with two amino groups in cis position, presenting values of $\angle \mathrm{N}-\mathrm{O}-\mathrm{S}$ angles smaller than those presented by the phosphate clamps.

In order to obtain some detailed information about the bonds and specially the non-covalent interaction a population analysis was performed using the NBO method using the same level of theory mentioned in methodology section. NBO shows all bonding orbitals and provides a clear view of charge donation throughout the platinum models studied, which includes phosphate clamps and arginine-forks. In Figure 9 one can find the orbitals involved with the studied hydrogen interactions that generates clamps and arginine-forks for the models shown in the Figure 5; it is not hard to notice the orbitals superposition that stabilizes the system. The orbitals involved in models 1 to 7 are presented as Supplementary Images (Figures S1, S2) (Zhurko, 2019).

The red arrows drawn on each picture illustrates the donation of a lone pair orbital to an antibonding one $\left(\mathrm{n} \rightarrow \mathrm{BD}^{*}\right)$. To sum up, all hydrogen interactions helped to stabilize the models suggested. When comparing both sulfates from HS and phosphates from DNA there are a slightly difference in energy that indicates the phosphate interactions are more stable.

The energy values for those superpositions have been reported on Table 4. The letters $x$ and $y$ (as displayed in Figure 6) are the 


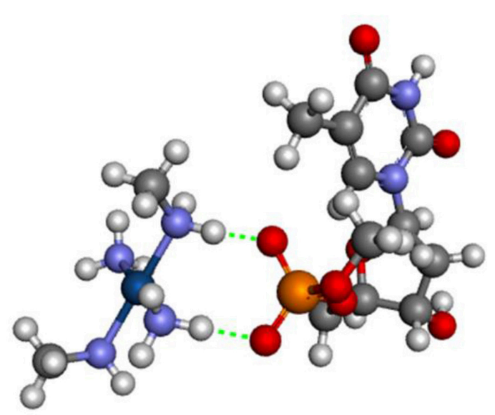

Model 1 - Phosphate Arginine-Fork

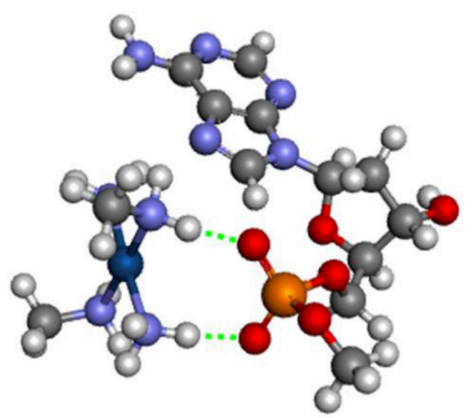

Model 2 - Phosphate Arginine-Fork

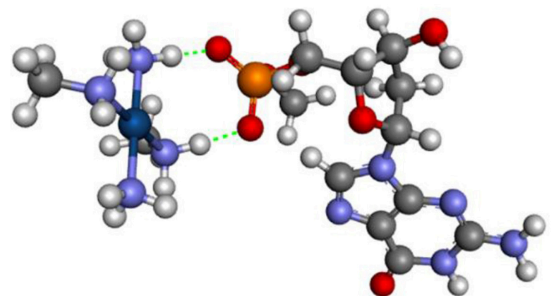

Model 3 - Phosphate Arginine-Fork

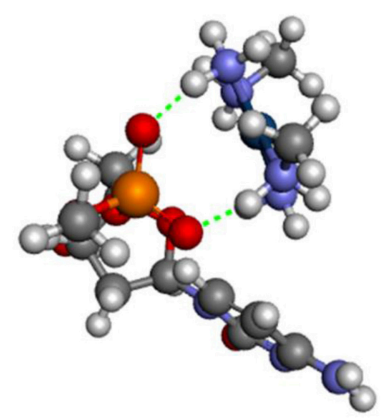

Model 4 - Phosphate Arginine-Fork

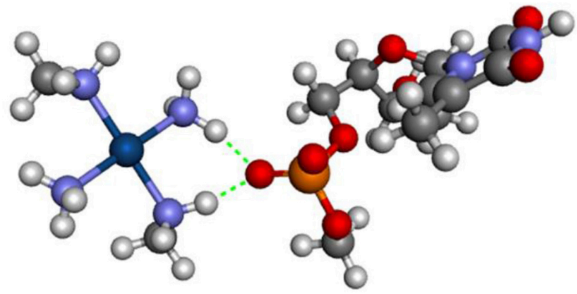

Model 1 - Phosphate Clamp

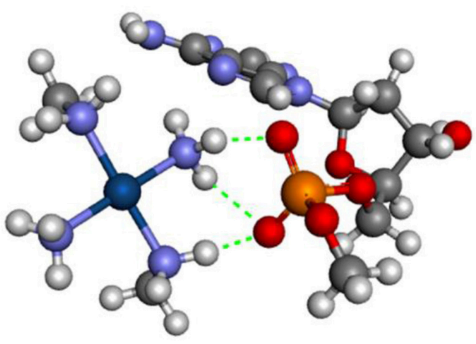

Model 2 - Phosphate Clamp

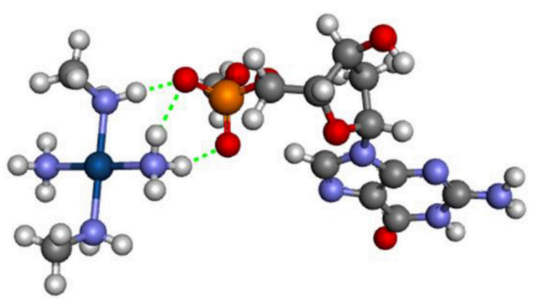

Model 3 - Phosphate Clamp

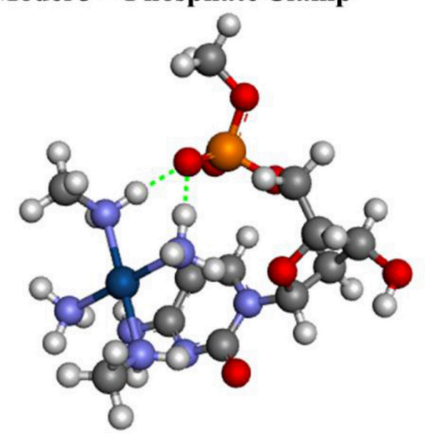

Model 4 - Phosphate Clamp

FIGURE 7 | Optimized structures of each different phosphate models 1-4 using the BHandH functional.

notation used to represent the interactions of hydrogen atoms from the $\mathrm{NH}_{3}$ and $\mathrm{NH}_{2}$ groups, respectively. Values correspond to the stabilizing energies, that is, in fact, how the formation of this interaction stabilizes the system itself. Because the clamp and fork structures are generated by the presence of the two interactions simultaneously one can be seen on the last column the summation of each bond contribution. The same table including the orbital occupation can be found in the Supporting Material (Table S1).

When comparing the stabilization energies for the clamps, it is visible that the phosphate one shows better stability than the sulfate, 70.03 and $53.97 \mathrm{kcal} \mathrm{mol}^{-1}$, respectively. The same pattern can be seen in the forks and specially in the arginine structure (in which the difference is up to $25 \mathrm{kcal} \mathrm{mol}^{-1}$ ). 

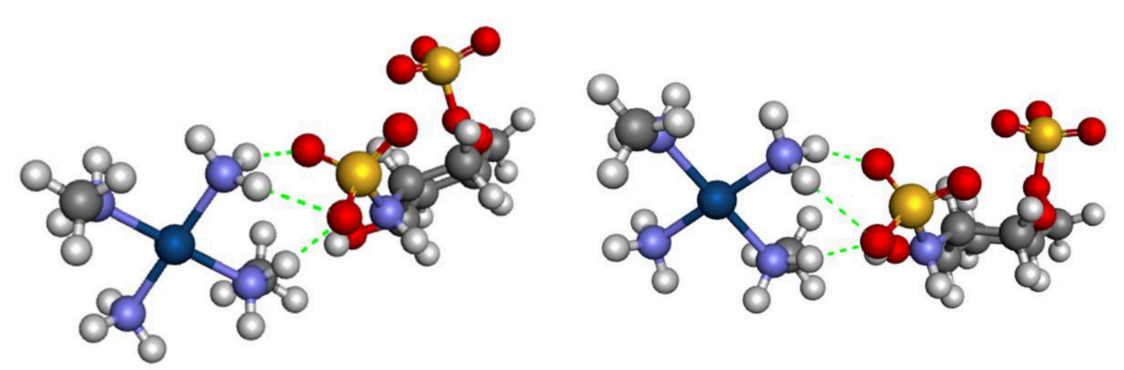

Model 5 - Sulfate Arginine-Fork
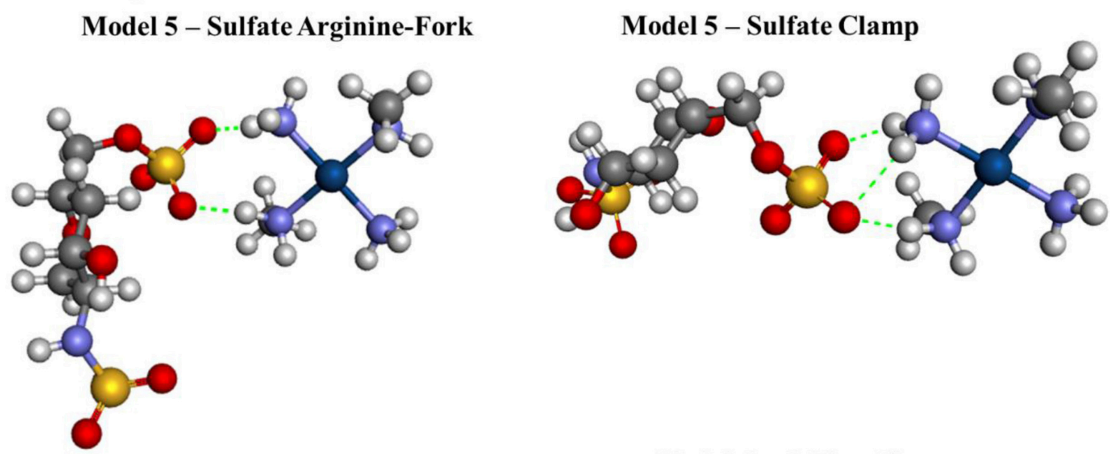

Model 6 - Sulfate Arginine-Fork
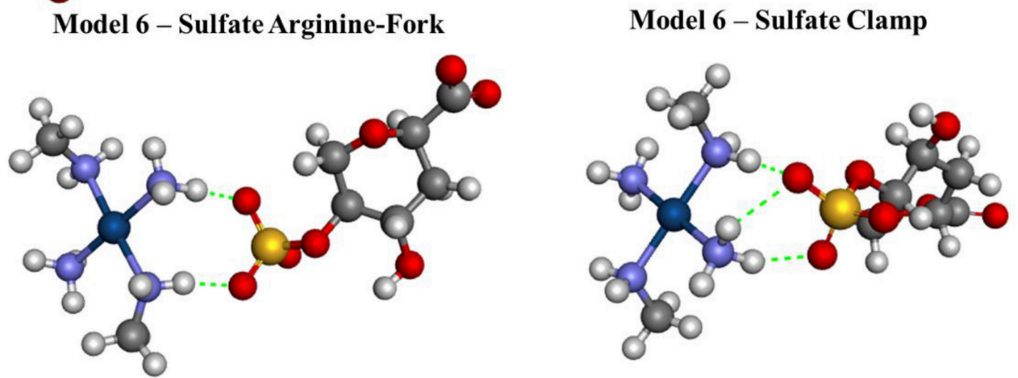

Model 7 - Sulfate Arginine-Fork

Model 7 - Sulfate Clamp

FIGURE 8 | Optimized structures of each different sulfate models 5-7 using the BHandH functional.

TABLE 3 | Distances (in Ångström) and bond angles (in degree) involved in the formation of clamps and arginine-forks.

\begin{tabular}{|c|c|c|c|c|c|c|c|}
\hline \multicolumn{2}{|l|}{ Models } & \multicolumn{2}{|c|}{ Distances/Å } & \multicolumn{4}{|c|}{ Angles $/^{\circ}$} \\
\hline & & $\mathbf{x}$ & y & $v_{1}$ & $v_{2}$ & $\omega_{1}$ & $\omega_{2}$ \\
\hline \multirow[t]{4}{*}{ Phosphate Arginine-fork } & 1 & 2.70 & 2.67 & 111.9 & 112.7 & 130.6 & 113.9 \\
\hline & 2 & 2.72 & 2.70 & 108.3 & 108.0 & 129.9 & 112.7 \\
\hline & 3 & 2.68 & 2.68 & 114.2 & 112.6 & 114.2 & 130.7 \\
\hline & 4 & 2.71 & 2.69 & 105.4 & 93.9 & 107.9 & 127.9 \\
\hline \multirow[t]{4}{*}{ Phosphate Clamp } & 1 & 2.71 & 2.70 & 100.8 & 100.4 & 135.3 & 134.3 \\
\hline & 2 & 2.92 & 2.72 & 105.4 & 99.2 & 128.9 & 90.9 \\
\hline & 3 & 2.85 & 2.71 & 105.9 & 102.3 & 125.7 & 92.8 \\
\hline & 4 & 2.76 & 2.71 & 102.3 & 100.7 & 126.3 & 124.5 \\
\hline \multirow[t]{3}{*}{ Sulfate Arginine-Fork } & 5 & 2.81 & 2.80 & 106.1 & 110.6 & 109.6 & 98.2 \\
\hline & 6 & 2.75 & 2.75 & 124.7 & 116.0 & 114.4 & 122.9 \\
\hline & 7 & 2.76 & 2.76 & 109.5 & 104.1 & 128.5 & 122.7 \\
\hline \multirow[t]{3}{*}{ Sulfate Clamp } & 5 & 2.89 & 2.80 & 105.9 & 102.9 & 109.5 & 94.6 \\
\hline & 6 & 2.89 & 2.79 & 104.5 & 101.1 & 113.0 & 94.5 \\
\hline & 7 & 2.85 & 2.79 & 105.5 & 103.6 & 122.2 & 97.1 \\
\hline Mean & & 2.79 & 2.73 & 107.9 & 104.9 & 121.1 & 111.3 \\
\hline
\end{tabular}

In the other hand, when comparing the phosphate ones with the platinum center it shows a slightly higher energy when forming clamps, contrarily to the sulfates that appears to be interacting more efficiently in a fork-shaped structure. In all studied models the oxygen's lone pair from the sulfate/phosphate to the antibonding orbital of the $\mathrm{N}-\mathrm{H}$ bond is clearly the primary donation. Such electron transfer is responsible for the interactions that stabilize all models and form the studied clamps and arginine-forks.

Figure 10 shows the optimized structure obtained for Models $\mathrm{A}, \mathrm{B}$, and $\mathrm{C}$ using the $\mathrm{BHand} \mathrm{H}$ functional. It is possible to observe the formation of the phosphate clamps and argininefork between the TriplatinNC complex and oxygen atoms from phosphate groups of two guanine nitrogen bases. The interactions of the type hydrogen bonds present in model A feature distances between the hydrogen and oxygen atoms ranges from 1.61 and $2.44 \AA$. For Model B and C, the distances are between 1.68 and $1.76 \AA$ and 1.63 and $2.29 \AA$, respectively. In Model A, a total of three phosphate clamps and two arginineforks were observed, and the $\mathrm{NH}_{3}$ groups of two Pt centers participate in both clamps and arginine-forks. Two structures of 


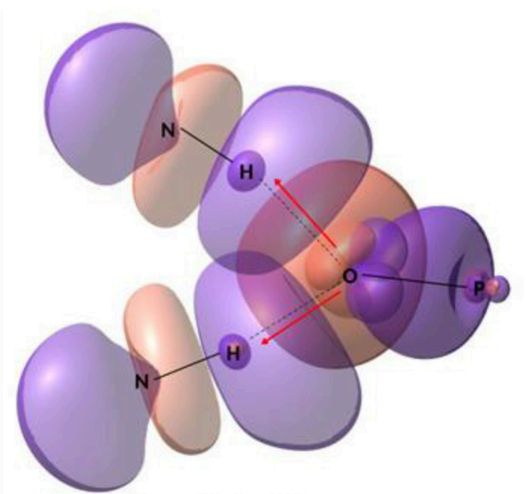

Phosphate Clamp

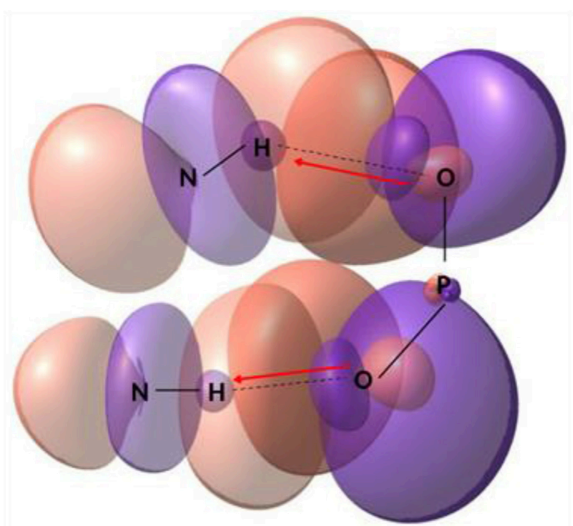

Phosphate Arginine-fork

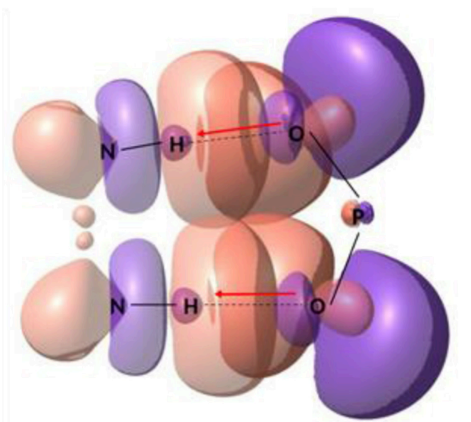

Arginine-fork ...Phosphate

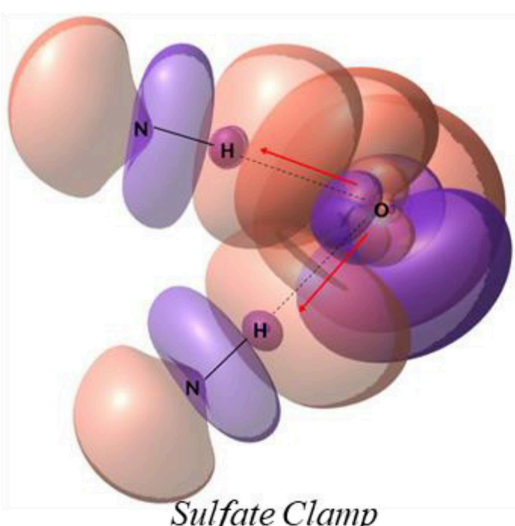

Sulfate Clamp

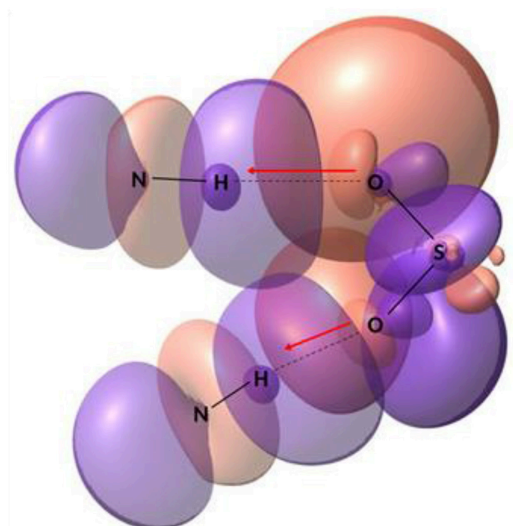

Sulfate Arginine-fork

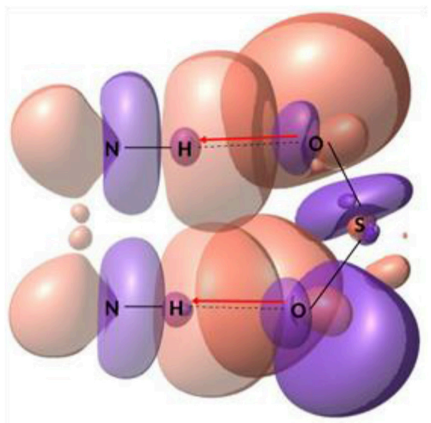

Arginine-fork ... Sulfate

FIGURE 9 | Natural bonding orbitals of models displayed on Figure 5. The electron donation is depicted by red arrows from oxygen atoms to N-H bond.

the phosphate clamps type were observed in the Model B. There was no formation of any arginine-fork structure in this model. The average distance between the oxygen atoms of the phosphate groups and the hydrogen atoms of the amino groups present in the $\mathrm{A}$ and $\mathrm{B}$ models is $1.83 \AA$. For Model C, the average distance between the hydrogen atoms of the amino groups and the oxygen atoms of the sulfate groups is $1.93 \AA$.

Model $\mathrm{C}$ showed a significantly higher number of noncovalent interactions compared to models $\mathrm{A}$ and $\mathrm{B}$. This difference can be justified by the presence of a higher number of sulfate groups in heparin compared to the number of phosphate groups present in the DNA in the models analyzed. According to Katner et al. (2018) heparin has a mean of 2.7 sulfate groups per disaccharide compared to the mean value of two phosphate groups per base pair present in DNA.

Table 5 shows the main values for distances and connection angles involved in the formation of the phosphate and sulfate clamps and arginine-forks. These calculated values are close to the experimental ones reported by Komeda et al. (2006) as can be seen in Table 5. The cis orientation of the amine groups is essential for the formation of the phosphate clamps and arginine-forks. It is noted that one of the phosphate groups 
defined in the high layer interacts with two platinum centers, forming two structures of phosphate clamps and two phosphate arginine-forks.

\section{CONCLUSION}

The study of hydrogen bond interactions by phosphate clamps and arginine-forks through mimetic models has been the main point described in this research paper. The mimetic models approach confirms that TriplatinNC complex has the capability to interact non-covalently with the phosphate groups present

TABLE 4 | Second-order perturbative energy values of donor-acceptor (bond-antibond) interactions from NBO calculations for the $\mathrm{H}$-bonds involved in the formation of clamps and Arginine-forks using the $\mathrm{BHandH}$ functional.

\begin{tabular}{lcccc}
\hline & & $\mathbf{E}_{\text {lig. } \mathbf{x}}$ & $\mathbf{E}_{\text {lig. } \mathbf{y}}$ & E $_{\text {TOTAL }}^{\mathbf{a}}$ \\
\hline Phosphate & Clamps & 33.94 & 36.09 & 70.03 \\
\multirow{2}{*}{ Sulfate } & Arginine-forks & 28.06 & 40.3 & 68.36 \\
& Clamps & 26.42 & 27.55 & 53.97 \\
\multirow{2}{*}{ Arginine-Forks } & Arginine-forks & 28.08 & 29.43 & 57.51 \\
& Phosphate & 43.82 & 45.18 & 89.00 \\
& Sulfate & 32.53 & 32.24 & 64.77 \\
\hline
\end{tabular}

All values are displayed in $\mathrm{kcal} \mathrm{mol}^{-1}$.

${ }^{a}$ The index $x$ and $y$ corresponds to each bond between oxygen and hydrogen atoms. Please, refer to Figure 6 for clarity.

${ }^{b} E_{T O T A L}$ corresponds to the summation of each column values. in the DNA and sulfate groups present in the HexaHS through the presence of the interactions such as phosphate clamps and arginine-forks that make possible the formation of a bidentate structure of the amino ligands and oxygen atoms. The interaction energies values suggest that the $\mathrm{BHandH}$ functional along with $6-31+\mathrm{G}(\mathrm{d}, \mathrm{p}) / \mathrm{SDD}$ basis sets provides the best values, confirming that such methodology is adequate for the analysis of the types of interaction studied in this work. NBO analysis proved efficient in describing the donation process for the models proposed here.

The structures obtained with a DNA fraction containing 6 nitrogenous base pairs and the TriplatinNC complex confirm the formation of the phosphate clamps through the formation of hydrogen interactions between the hydrogen atoms from amine groups in the cis position of the platinum center, with the same oxygen atom of a phosphate group present in the DNA strand. Argininefork structures have also been observed and have similar structures to clamps. These interactions contribute to complex-DNA interaction.

It is known that molecules such as cisplatin can find different biomolecules in which it is possible to form bonds, thus presenting a competition of several molecules to each other, affecting bioavailability, with only a small fraction of the compound effectively binding to DNA. The results shown in this work suggest that non-covalent interactions can occur with other biomolecules other than DNA, so that the similarity of sulfate groups present in heparin with the phosphate groups of DNA allows the interaction of the TriplatinNC complex

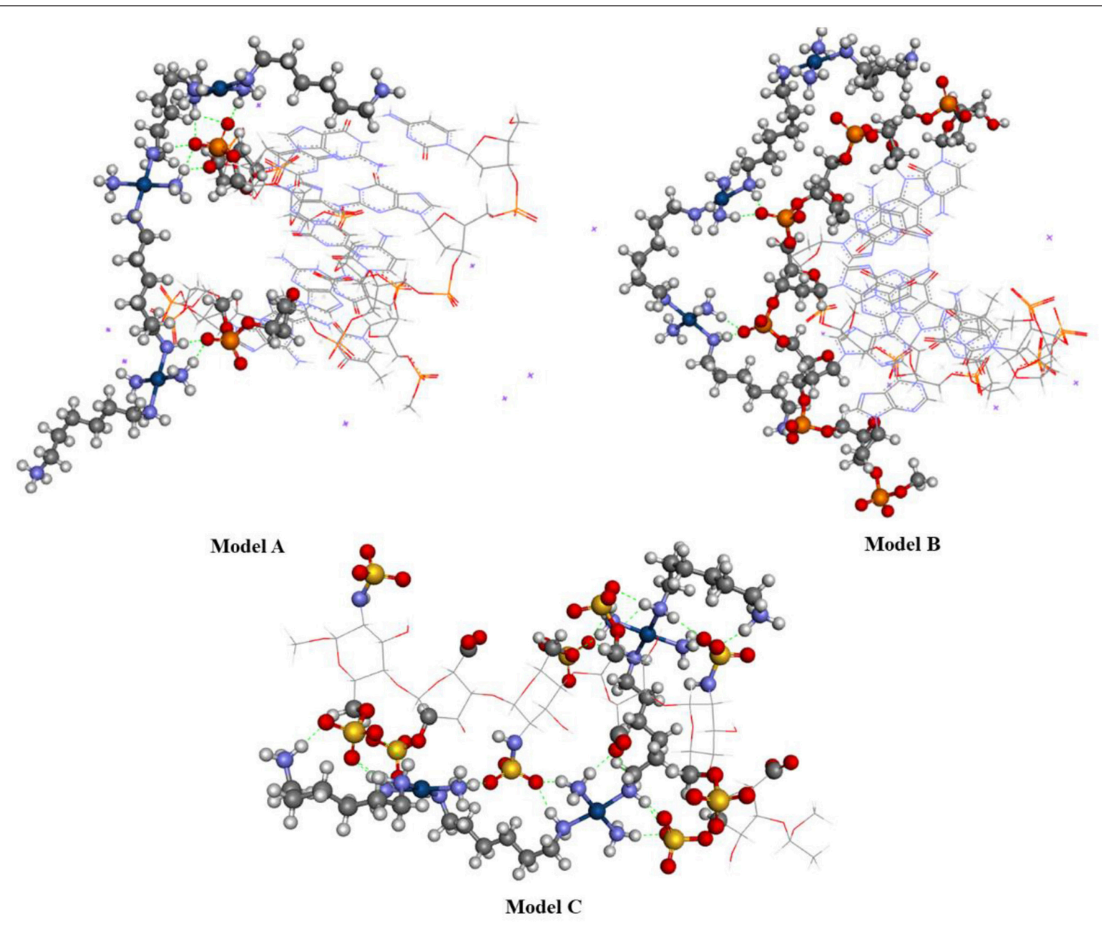

FIGURE 10 | Optimized structure of models (A-C) using the functional BHandH. 
TABLE 5 | Distances (in Ångström) and bond angles (in degree) involved in the formation of non-covalent interactions for models A-C.

\begin{tabular}{|c|c|c|c|c|c|c|c|}
\hline & \multirow[b]{2}{*}{$\mathbf{x}$} & \multicolumn{2}{|c|}{ Distances/Å } & \multicolumn{4}{|c|}{ Angles $/^{\circ}$} \\
\hline & & $\mathbf{y}$ & $v_{1}$ & $v_{2}$ & $\omega_{1}$ & $\omega_{2}$ & \\
\hline \multirow[t]{5}{*}{ Model A } & $P C 1^{a}$ & 2.64 & 2.99 & 91.4 & 102.5 & 99.3 & 138.7 \\
\hline & $P A F 1^{b}$ & 2.64 & 2.83 & 113.2 & 102.5 & 106.4 & 138.7 \\
\hline & PC2 & 2.72 & 2.75 & 103.3 & 104.3 & 126.5 & 111.4 \\
\hline & PAF2 & 3.03 & 2.75 & 103.3 & 105.9 & 126.5 & 97.9 \\
\hline & PC3 & 2.73 & 2.73 & 101.5 & 101.8 & 121.5 & 127.7 \\
\hline \multirow[t]{2}{*}{ Model B } & PC1 & 2.72 & 2.76 & 102.6 & 104.1 & 167.4 & 118.2 \\
\hline & PC2 & 2.69 & 2.76 & 100.3 & 102.3 & 111.6 & 155.8 \\
\hline \multirow[t]{12}{*}{ Model C } & $\mathrm{SC}_{1}{ }^{\mathrm{C}}$ & 2.89 & 2.86 & 102.0 & 101.0 & 96.5 & 138.4 \\
\hline & $\mathrm{SAF}_{1}{ }^{\mathrm{d}}$ & 2.89 & 2.87 & 128.7 & 101.0 & 96.8 & 138.4 \\
\hline & SC2 & 2.80 & 2.95 & 97.6 & 102.7 & 115.3 & 109.1 \\
\hline & SC3 & 2.78 & 2.75 & 102.5 & 101.7 & 140.2 & 148.6 \\
\hline & SC4 & 2.76 & 2.82 & 92.3 & 94.7 & 147.6 & 141.9 \\
\hline & SC5 & 2.86 & 3.02 & 97.0 & 101.4 & 97.7 & 125.6 \\
\hline & SAF2 & 2.86 & 2.93 & 108.2 & 101.4 & 101.9 & 125.3 \\
\hline & SC6 & 2.80 & 2.92 & 96.5 & 100.2 & 155.9 & 135.4 \\
\hline & SC7 & 2.89 & 2.94 & 103.9 & 105.9 & 110.4 & 101.3 \\
\hline & SAF3 & 2.89 & 3.04 & 85.7 & 105.9 & 101.8 & 101.3 \\
\hline & SC8 & 2.86 & 2.78 & 105.8 & 103.5 & 117.9 & 106.3 \\
\hline & SAF4 & 2.86 & 2.78 & 105.8 & 118.8 & 117.9 & 101.7 \\
\hline Exp. & $\mathrm{PC}_{\text {mean }}$ & 2.90 & 2.95 & 103 & 100 & 127 & 140 \\
\hline
\end{tabular}

aPC, phosphate clamp.

${ }^{b} P A F$, phosphate arginine-fork.

¿SC, sulfate clamp.

dSAF, sulfate arginine-fork.

with the heparin. The size of the heparin model used was shown to be efficient interacting with the three platinum centers present in TriplatinNC. The formation of eight sulfate clamps structures and four arginine-fork structures were noted in this work.

\section{REFERENCES}

Andrae, D., Häußermann, U., Dolg, M., Stoll, H., and Preuß, H. (1990). Energyadjusted ab initio pseudopotentials for the second and third row transition elements. Theor. Chim. Acta 77, 123-141.

Barry, N. P., and Sadler, P. J. (2013). Exploration of the medical periodic table: towards new targets. Chem. Commun. 49, 5106-5131. doi: 10.1039/ c3cc $41143 \mathrm{e}$

Becke, A. D. (1993a). Density-functional thermochemistry. III. The role of exact exchange. J. Chem. Phys. 98, 5648-5652. doi: 10.1063/1.464913

Becke, A. D. (1993b). A new mixing of Hartree-Fock and local density-functional theories. J. Chem. Phys. 98, 1372-1377. doi: 10.1063/1.464304

Benedetti, B. T., Peterson, E. J., Kabolizadeh, P., Martínez, A., Kipping, R., and Farrell, N. P. (2011). Effects of noncovalent platinum drugprotein interactions on drug efficacy: use of fluorescent conjugates as probes for drug metabolism. Mol. Pharm. 8, 940-948. doi: 10.1021/ mp2000583

Boulikas, T., and Vougiouka, M. (2003). Cisplatin and platinum drugs at the molecular level. Oncol. Rep. 10, 1663-1682. doi: 10.3892/or.10.6.1663

Chen, B., and Zhou, L. (2015). Computational study on mechanisms of the anticancer drug: cisplatin and novel polynuclear platinum(II) interaction with sulfur-donor biomolecules and DNA purine bases.

\section{AUTHOR CONTRIBUTIONS}

NMPR conducted the simulations of the structures of the phosphate groups. FHCF conducted the simulations of the structures of the sulfate groups. FHCF and LASC conducted the NBO analyzes. NMPR and FHCF obtained the structures using the ONIOM methodology. NMPR, FHCF, NPF, and LASC participated in the discussion of the results. NMPR, FHCF, and LASC wrote the manuscript together.

\section{FUNDING}

LASC is also a member of the Rede Mineira de Química (RQMG), supported by FAPEMIG (Project: CEX-RED-00010-14). This project has also been partly supported by pilot funding from the Massey Cancer Center, VCU, with funding, in part, from NIH-NCI Cancer. Center Support Grant P30 CA016059.

\section{ACKNOWLEDGMENTS}

LASC would like to acknowledge the $\mathrm{CNPq}$ (Conselho Nacional de Desenvolvimento Científico e Tecnológico) for the research grant. NMPR would like to acknowledge CAPES and UFJF for her Masters' scholarship. NMPR is now on her Doctorate which is also supported by CAPES. FHCF would like to thanks UFJF for his scientific initiation scholarship.

\section{SUPPLEMENTARY MATERIAL}

The Supplementary Material for this article can be found online at: https://www.frontiersin.org/articles/10.3389/fchem. 2019.00307/full\#supplementary-material

Data Sheet S1 | Figures S1 and S2 show NBO of models 1-7. Table S1 shows details about NBO calculations. Spatial coordinates (xyz) of the optimized structures are available.

Comput. Theor. Chem. 1074, 36-49. doi: 10.1016/j.comptc.2015. 09.023

Chung, L. W., Sameera, W. M. C., Ramozzi, R., Page, A. J., Hatanaka, M., and Petrova, G. P. (2015). The ONIOM method and its applications. Chem. Rev. 115, 5678-5796. doi: 10.1021/cr5004419

Esteves, L. F., Santos, H. F., and Costa, L. A. S. (2015). The conformation effect of the diamine bridge on the stability ofdinuclear platinum(II) complexes and their hydrolysis. J. Mol. Graph. Model. 61, 290-296. doi: 10.1016/j.jmgm.2015.08.006

Farrell, N. P. (2015). Multi-platinum anti-cancer agents. Substitution-inert compounds for tumor selectivity and new targets. Chem. Soc. Rev. 44, 8773-8785. doi: 10.1039/c5cs00201j

Frisch, M. J., Trucks, G. W., Schlegel, H. B., Scuseria, G. E., Robb, M. A., Cheeseman, J. R., et al. (2016). Gaussian 09, Revision A.02. Wallingford, CT: Gaussian, Inc.

Gkionis, K., Mutter, S. T., and Platts, J. A. (2013). QM/MM description of platinum-DNA interactions: comparison of binding and DNA distortion of five drugs. R. Soc. Chem. Adv. 3, 4066-4073. doi: 10.1039/ c3ra23041d

Gkionis, K., and Platts, J. A. (2012). QM/MM studies of cisplatin complexes with DNA dimer and octamer. Comput. Theor. Chem. 993, 60-65. doi: 10.1016/j.comptc.2012.05.034 
Harris, A. L., Yang, X., Hegmans, A., Povirk, L., Ryan, J. J., Kelland, L., et al. (2005). Synthesis, characterization, and cytotoxicity of a novel highly charged trinuclear platinum compound. Enhancement of cellular uptake with charge inorganic. Chem. Commun. 44, 9598-9600. doi: 10.1021/ic051390z

Hay, P. J., and Wadt, W. R. (1985a). Ab initio effective core potentials for molecular calculations. Potentials for the transition metal atoms Sc to Hg. J. Chem. Phys. 82, 270-283. doi: 10.1063/1.448799

Hay, P. J., and Wadt, W. R. (1985b). Ab initio effective core potentials for molecular calculations. Potentials for main group elements Na to Bi. J. Chem. Phys. 82, 284-298. doi: 10.1063/1.448800

Hay, P. J., and Wadt, W. R. (1985c). Ab initio effective core potentials for molecular calculations. Potentials for $\mathrm{K}$ to Au including the outermost core orbitals. J. Chem. Phys. 82, 299-310. doi: 10.1063/1.448975

Heerdt, G., and Morgon, N. H. (2011). Validação computacional de métodos compostos no estudo de propriedades moleculares. Química Nova 34, 868-873. doi: 10.1590/S0100-40422011000500024

Hegmans, A., Berners-Price, S. J., Davies, M. S., Thomas, D. S., Humphreys, A. S., and Farrell, N. P. (2004). Long range 1,4 and 1,6-interstrand cross-links formed by a trinuclear platinum complex. Minor groove preassociation affects kinetics and mechanism of cross-link formation as well as adduct structure. J. Am. Chem. Soc. 126, 2166-2180. doi: 10.1021/ja036105u

Here, W. J., Ditchfield, R., and Pople, J. A. (1972). Self-consistent molecular orbital methods. XII. Further extensions of Gaussian-type basis sets for use in molecular orbital studies of organic molecules. J. Chem. Phys. 56, 2257-2261. doi: 10.1063/1.1677527

Kasparkova, J., Zehnulova, J., Farrell, N. P., and Brabec, V. (2002). DNA interstrand cross-links of the novel antitumor trinuclear platinum complex BBR3464. J. Biol. Chem. 277, 48076-48086. doi: 10.1074/jbc.M208016200

Katner, S. J., Johnson, W. E., Peterson, E. J., Page, P., and Farrell, N. P. (2018). Comparison of metal-ammine compounds binding to DNA and Heparin. Glycans as ligands in bioinorganic chemistry. Inorg. Chem. 57, 3116-3125. doi: 10.1021/acs.inorgchem.7b03043

Komeda, S., Moulaei, T., Chikuma, M., Odani, A., Kipping, R., Farrell, N. P., et al. (2010). The phosphate clamp: a small and independent motif for nucleic acid backbone recognition. Nucleic Acids Res. 39, 325-336. doi: 10.1093/nar/g kq723

Komeda, V., Moulaei, T., Woods, K. K., Chikuma, M., Farrell, N. P., and Williams, L. D. (2006). A third mode of DNA binding: phosphate clamps by a polynuclear platinum complex. J. Am. Chem. Soc. 128, 16092-16103. doi: 10.1021/ja06 2851y

Malina, J., Farrell, N. P., and Brabec, V. (2014). DNA condensing effects and sequence selectivity of DNA binding of antitumor noncovalent polynuclear platinum complexes. Inorg. Chem. 53, 1662-1671. doi: 10.1021/ic40 $2796 \mathrm{k}$

Mangrum, J. B., and Farrell, N. P. (2010). Excursions in polynuclear platinum DNA binding. Chem. Commun. 46, 6640-6650. doi: 10.1039/c0cc01254h

Manzotti, C., Pratesi, G., Menta, E., Di Domenico, R., Cavalletti, E., Fiebig, H. H., et al. (2000). BBR 3464: a novel triplatinum complex, exhibiting a preclinical profile of antitumor efficacy different from cisplatin. Clin. Cancer Res. 6, 2626-2634.

Morokuma, K. (2003). ONIOM and its applications to material chemistry and catalyses. Bull. Korean Chem. Soc. 24, 797-801. doi: 10.5012/bkcs.2003.24.6.797

Mulloy, B., Forster, M., Jones, C., and Davies, D. B. (1993). N.m.r. and molecularmodelling studies of the solution conformation of heparin. Biochem. J. 293, 849-858. doi: 10.1042/bj2930849

Olszewski, U., and Hamilton, G. (2010). A better platinum-based anticancer drug yet to come? Anticancer Agents Med. Chem. 10, 293-301. doi: $10.2174 / 187152010791162306$
Paixão, N. M., Esteves, L. F., Anconi, C. P. A., Nascimento, C. S. Jr., Almeida, W. B., Santos, H. F., et al. (2012). Theoretical study of inclusion of a dinuclear platinum(II) complex in $\alpha, \beta$ and $\gamma$-cyclodextrins. Int. J. Quant. Chem. 112, 3403-3408. doi: 10.1002/qua.24271

Peterson, E. J., Gerard Daniel, A., Katner, S. J., Bohlmann, L., Chang, C. W., Bezos, A., et al. (2017). Antiangiogenic platinum through glycan targeting. Chem. Sci. 8, 241-252. doi: 10.1039/c6sc02515c

Peterson, E. J., Menon, V. R., Gatti, L., Kipping, R., Dewasinghe, D., Perego, P., et al. (2014). Nucleolar targeting by platinum: p53-independent apoptosis follows rRNA inhibition, cell-cycle arrest, and DNA compaction. Mol. Pharm. 12, 287-297. doi: 10.1021/mp5006867

Prisecaru, A., Molphy, Z., Kipping, R. G., Peterson, E. J., Qu, Y., Kellett, A., et al. (2014). The phosphate clamp: sequence selective nucleic acid binding profiles and conformational induction of endonuclease inhibition by cationic triplatin complexes. Nucleic Acid Res. 42, 13474-13487. doi: 10.1093/nar/ gku1157

Qu, Y., Harris, A., Hegmans, A., Petz, A., Kabolizadeh, P., Penazova, H., et al. (2004). Synthesis and DNA conformational changes of noncovalent polynuclear platinum complexes. J. Inorg. Biochem. 98, 1591-1598. doi: 10.1016/j.jinorgbio.2004.07.012

Qu, Y., Kipping, G. R., and Farrel, N. P. (2015). Solution studies on DNA interactions of substitution-inert platinum complexes mediated via the phosphate clamp. Dalton Trans. 44, 3563-3572. doi: 10.1039/ c4dt03237c

Rappé, A. K., Casewit, C. J., Colwell, K. S., Goddard, W. A. III., and Skiff, W. M. (1992). UFF, a full periodic table force field for molecular mechanics and molecular dynamics simulations. J. Am. Chem. Soc. 114, 10024-10035. doi: 10.1021/ja00051a040

Riccardi, A., Meco, D., Ferlini, C., Servidei, T., Carelli, G., Segni, G., et al. (2001). In vitro and in vivo antitumor activity of the novel trinuclear platinum complex BBR 3464 in neuroblastoma. Cancer Chemother. Pharmacol. 47, 498-504. doi: $10.1007 / \mathrm{s} 002800000264$

Scalmani, G., and Frisch, M. J. (2010). Continuous surface charge polarizable continuum models of solvation. I. General formalism. J. Chem. Phys. 132:114110. doi: 10.1063/1.3359469

Wang, K., and Gao, E. (2014). Recent advances in multinuclear complexes as potential anticancer and DNA binding agents. Anticancer Agents Med. Chem. 14, 147-169. doi: 10.2174/18715206113139990313

Wheate, N. J., and Collins, J. G. (2005). Multi-nuclear platinum drugs: a new paradigm in chemotherapy. Curr. Med. Chem. Anticancer Agents 5, 267-279. doi: 10.2174/1568011053765994

Wong, E., and Giandomenico, C. M. (1999). Current status of platinum-based antitumor drugs. Chem. Rev. 99, 2451-2466. doi: 10.1021/cr980420v

Zhurko, G. A. (2019). Chemcraft - Graphical Program for Visualization of Quantum Chemistry Computations, Version 1.8. Ivanovo. Available online at: https:// chemcraftprog.com

Conflict of Interest Statement: The authors declare that the research was conducted in the absence of any commercial or financial relationships that could be construed as a potential conflict of interest.

Copyright (c) 2019 Rosa, Ferreira, Farrell and Costa. This is an open-access article distributed under the terms of the Creative Commons Attribution License (CC BY). The use, distribution or reproduction in other forums is permitted, provided the original author(s) and the copyright owner(s) are credited and that the original publication in this journal is cited, in accordance with accepted academic practice. No use, distribution or reproduction is permitted which does not comply with these terms. 